\title{
Ultrafast dynamics of coherent optical phonons and nonequilibrium electrons in transition metals
}

\author{
Muneaki Hase, ${ }^{1, *}$ Kunie Ishioka,,${ }^{1,4}$ Jure Demsar, ${ }^{2}$ Kiminori Ushida, ${ }^{3}$ and Masahiro Kitajima ${ }^{1,4}$ \\ ${ }^{1}$ Materials Engineering Laboratory, National Institute for Materials Science, 1-2-1 Sengen, Tsukuba, 305-0047, Japan \\ ${ }^{2}$ Department for Complex Matter, J. Stefan Institute, Jamova 39, Ljubljana, SI-1000, Slovenia \\ ${ }^{3}$ RIKEN (The Institute of Physical and Chemical Research), 2-1 Hirosawa, Wako, 351-0198, Japan \\ ${ }^{4}$ Graduate School of Pure and Applied Sciences, University of Tsukuba, 1-2-1 Sengen, Tsukuba, Ibaraki 305-0047, Japan
}

(Received 21 October 2004; published 6 May 2005)

\begin{abstract}
The femtosecond optical pump-probe technique was used to study dynamics of photoexcited electrons and coherent optical phonons in transition metals $\mathrm{Zn}$ and $\mathrm{Cd}$ as a function of temperature and excitation level. The optical response in time domain is well fitted by linear combination of a damped harmonic oscillation because of excitation of coherent $E_{2 g}$ phonon and a subpicosecond transient response due to electron-phonon thermalization. The electron-phonon thermalization time monotonically increases with temperature, consistent with the thermomodulation scenario, where at high temperatures the system can be well explained by the twotemperature model, while below $\approx 50 \mathrm{~K}$ the nonthermal electron model needs to be applied. As the lattice temperature increases, the damping of the coherent $E_{2 g}$ phonon increases, while the amplitudes of both fast electronic response and the coherent $E_{2 g}$ phonon decrease. The temperature dependence of the damping of the $E_{2 g}$ phonon indicates that population decay of the coherent optical phonon due to anharmonic phonon-phonon coupling dominates the decay process. We present a model that accounts for the observed temperature dependence of the amplitude assuming the photoinduced absorption mechanism, where the signal amplitude is proportional to the photoinduced change in the quasiparticle density. The result that the amplitude of the $E_{2 g}$ phonon follows the temperature dependence of the amplitude of the fast electronic transient indicates that under the resonant condition both electronic and phononic responses are proportional to the change in the dielectric function.
\end{abstract}

DOI: 10.1103/PhysRevB.71.184301

PACS number(s): 78.47.+p, 78.20.-e, 63.20.Kr, 78.66.Bz

\section{INTRODUCTION}

Dynamics of nonequilibrium electrons and phonons in metals, semiconductors, and superconductors have been the focus of much attention because of their fundamental interest in solid-state physics. In particular, electron-phonon interaction is the center of the interest for understanding the nature of nonequilibrium electron relaxation [electron-phonon $(e-p h)$ thermalization], of dressed electrons (an electron plus its phonon cloud), of phonon self-energy (real part corresponds to the change in phonon frequency), and of phononinduced electron-electron interaction (this effect gives the birth of the Cooper pair in superconductors). ${ }^{1}$

In metals, relaxation dynamics of optically excited nonequilibrium electrons has been extensively studied by transient-reflectivity (TR) or transient-transmission (TT) pump-probe techniques using femtosecond lasers. ${ }^{2-6}$ The $e$-ph thermalization occurs on a subpicosecond time scale in metals and has been traditionally described by the use of the two-temperature model (TTM) ${ }^{6-9}$ Besides the observation of the dynamics of nonequilibrium electrons, coherent acousticphonon pulses have been investigated by using the pumpprobe angular-deflection technique. ${ }^{10,11}$ The acoustic-phonon pulse is generated through transient heating of the sample surface induced by excitation with intense laser pulses. The response from acoustic-phonon pulses is typically observed on a several hundred picosecond time scale (or gigahertz frequency range). ${ }^{12}$ Since the electron-phonon thermalization that we are considering takes place on a subpicosecond time scale (or terahertz frequency range), ${ }^{6}$ coherent optical phonons in metals, which are impulsively excited and decay within a few picoseconds, may play an important role in relaxation of hot electrons.

Recently, Melnikov et al. have observed the surface coherent optical phonon $(\omega=2.9 \mathrm{THz})$ at the surface of $\mathrm{Gd}$ metal using the second-harmonic generation (SHG) technique. ${ }^{13}$ They focused their attention into the generation mechanism of the coherent optical phonon and coupled coherent magnon. The surface coherent optical phonon was excited by a transient charge separation at the surface, which is similar proposal to the displacive excitation mechanism (DECP). ${ }^{14}$ Furthermore, Bovensiepen et al. ${ }^{15}$ studied both surface and bulk coherent optical phonons in Gd metal using the SHG and TR techniques, respectively. They discussed the transient frequency shifts observed for both phonons. Watanabe et al. revealed coherent surface vibration on Cs/Pt(111) system by using the SHG technique. ${ }^{16}$ The coherent stretching vibration $(\omega=2.2 \mathrm{THz})$ was generated by resonant-impulsive stimulated Raman scattering (ISRS), ${ }^{17}$ and the damping of the coherent surface vibration was dominated by pure dephasing caused by hot electrons at absorbate. Thus, the SHG technique has mainly enabled studying surface coherent optical phonons on metallic surfaces. Nevertheless, investigations of bulk coherent optical phonons in metals using conventional TR or TT pump-probe techniques are still few mainly because of the very short optical penetration depth in metals stemming from the absorption by free electrons (Drude absorption). Therefore the change in the refractive index associated with the coherent phonon oscillations are very weak in the bulk, making it very difficult to 
measure. Although study of the surface coherent optical phonon in metal surfaces offers interesting applications, e.g., controlling chemical reactions on metal surfaces, there is also tremendous motivation to observe and control the bulk coherent optical phonon in metals in order to study transient melting as a precursor of phase transition, ${ }^{18}$ as well as controlling electron-phonon scattering. ${ }^{19}$

In absorbing media, e.g., in semimetals, resonant ISRS has been proposed as the main generation mechanism of the coherent $\mathrm{A}_{1 g}$ phonon. ${ }^{17,20}$ Stevens et al. considered both the standard Raman susceptibility and the electrostrictive tensor as the driving force for resonant ISRS. ${ }^{20}$ Since electrostrictive force is proportional to the dielectric function, the amplitude of the coherent phonon depends on the incident photon energy. ${ }^{20}$ In some metals, interband transition occurs in the near infrared region, which appears as a peak in the imaginary part of the dielectric function, and the interband transition could enhance the amplitude of the coherent phonons through the resonant ISRS process. Therefore the realization of generation and observation of bulk coherent optical phonons in metals using the TR pump-probe technique requires (i) existence of $k=0$ optical phonon, which can be observed by Raman scattering, and (ii) a spectral peak in the imaginary part of the dielectric function, which is related to interband transition. Appropriate candidates of the metallic samples that meet the above requirements are $\mathrm{Zn}$, $\mathrm{Cd}$, and $\mathrm{Mg}$, in which $k=0$ optical phonon modes were, in fact, observed in Raman scattering. ${ }^{21}$

In this paper, we report on the observation of both bulk coherent optical phonon and nonequilibrium electrons in $\mathrm{Zn}$ and $\mathrm{Cd}$ using a femtosecond pump-probe reflectivity technique with high sensitivity of $\Delta R / R=10^{-7}$. The coherent $E_{2 g}$ phonon, whose oscillatory frequency is in the terahertz region, is generated by optical excitation of nonequilibrium electrons (interband transition). The amplitude of the oscillations decreases dramatically as the temperature is raised from $7 \mathrm{~K}$ to room temperature. Importantly, the temperature dependence of the amplitude of oscillation closely follows the temperature dependence of the subpicosecond electronic transient, suggesting that under the resonant condition both electronic and phononic responses are proportional to the change in the dielectric function. The relaxation time of the fast electronic response decreases with decreasing temperature, showing saturation at temperatures below $100 \mathrm{~K}$. In fact, this behavior closely follows the temperature dependence of the electron-phonon thermalization time observed in simple metals, such as Au, Ag. ${ }^{9}$ In order to explain the peculiar temperature dependence of the amplitude of the electronic transient, we have derived a simple model, where the photoinduced change in reflectivity is governed by photoinduced absorption, where the initial states lie near the Fermi energy. Assuming the so-called two-temperature model $^{9}$ and energy conservation law we were able to reproduce the temperature dependence of the transient amplitude extremely well-further supporting the association of a fast transient with electron-phonon thermalization and correlation between electronic and phononic responses via the dielectric function. We have extended the range of pump fluences up to $6 \mathrm{~mJ} / \mathrm{cm}^{2}$ and study phonon softening as a spectator of thermal expansion. In Sec. V, we discuss the temperature depen-

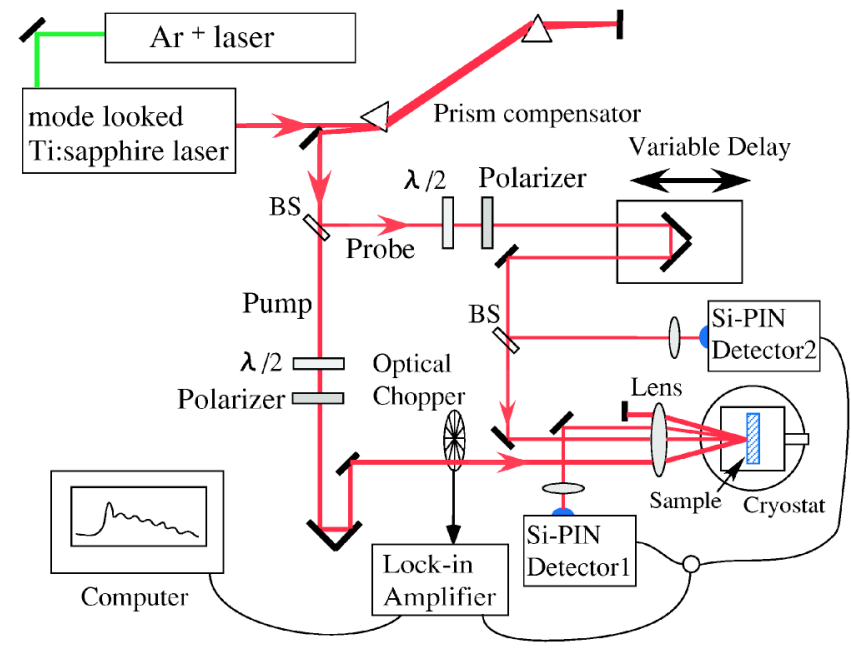

FIG. 1. (Color online) Schematics of femtosecond pump-probe experiment. BS: beam splitter, $\lambda / 2$ : half wave plate. The difference of the photocurrent of the two Si-PIN detectors was amplified and thus reduced current noise from the laser source.

dence of the amplitude of the coherent optical phonons in $\mathrm{Zn}$ in terms of Stevens' model. ${ }^{20}$

\section{EXPERIMENTAL TECHNIQUE}

The samples used here were single crystals of $\mathrm{Zn}$ and $\mathrm{Cd}$ with cut and polished (0001) surfaces. The femtosecond pump-probe measurements (Fig. 1) were carried out in a temperature range from 7 to $295 \mathrm{~K}$ using a cryostat. The light source used was a mode-locked Ti:sapphire laser whose pulse width was $\sim 20 \mathrm{fs}$ as determined by autocorrelation of $\sim 30 \mathrm{fs},{ }^{22}$ and the repetition rate of $87 \mathrm{MHz}$. The pump-beam power was varied from 20 to $120 \mathrm{~mW}$, while the probebeam power was fixed at $5 \mathrm{~mW}$. The polarizations of the pump and probe beams are orthogonal to each other to avoid picking the scattered pump beam on the detector. We used a lock-in detection where the pump-beam was mechanically chopped at $2 \mathrm{kHz}$. Both pump- and probe-beams were focused onto a diameter of $\sim 100 \mu \mathrm{m}$ on the sample. The penetration depth of the laser light with a wavelength of $800 \mathrm{~nm}$ $(=1.55 \mathrm{eV})$ was estimated to be $\sim 13 \mathrm{~nm}$ based on the absorption coefficient of $7.59 \times 10^{5} \mathrm{~cm}^{-1}$. Therefore the contribution to the signal from the surface oxide layer is negligibly small. At the pump fluence of $9.2 \mu \mathrm{J} / \mathrm{cm}^{2}(60 \mathrm{~mW})$ we estimated the maximum electron temperature rise $\Delta T_{e}^{m}$ (assuming a thermalized electron gas) to be $\leqslant 100 \mathrm{~K}$ at $7 \mathrm{~K} \cdot{ }^{23}$ Thus the experiments can be considered as being in the reasonably weak excitation regime. ${ }^{8,24}$ The TR signal $(\Delta R / R)$ was measured to extract the relaxation dynamics of nonequilibrium carriers and the bulk coherent optical phonon as a function of the time delay after excitation pulse. ${ }^{25}$

\section{GENERATION OF COHERENT OPTICAL PHONONS}

Traditionally, generation of coherent optical phonons has been described by either $\mathrm{DECP}^{14}$ (for absorbing media) or ISRS $^{26}$ (for transparent media). In DECP mechanism, photo- 
excitation induces changes in the electronic energydistribution function, and as a result, the crystal lattice begins to oscillate around the new equilibrium position; $Q_{0}(t)$ $=\kappa n(t)$, where $\kappa$ is a constant and $n(t)$ is the photoexcited carrier density. ${ }^{14}$ In the first order only the $\mathrm{A}_{1 g}$ totally symmetric modes are coherently excited by the DECP mechanism. Recently, Stevens et al. have suggested that the combination of DECP and ISRS is possible in semimetals. We briefly introduce their model below. ${ }^{20}$

The impulsive stimulated Raman scattering equation of motion for an optical vibrational mode whose normal coordinate is labeled $Q$ is given by ${ }^{20,26}$

$$
\frac{\partial^{2} Q}{\partial t^{2}}+2 \Gamma \frac{\partial Q}{\partial t}+\Omega_{0}^{2} Q=F(t) \equiv \frac{N v_{c}}{2} \sum_{k, l} R_{k l} E_{k} E_{l}
$$

where $\Gamma$ and $\Omega_{0}$ are the vibrational damping and the frequency, respectively, $N$ is the number of cells, $v_{c}$ is the volume of the unit cell, while $E_{k, l}$ are components of the electric field delivered by the broad spectrum of the ultrashort pump light. According to wave-vector and energy-conservation rules, a phonon mode with frequency $\omega_{l}-\omega_{k}=\Omega_{0}$ and wave vector $k_{l}-k_{k}=k_{0}$ can be excited. ${ }^{25,26}$ The Raman tensor $R_{k l}$ denotes both the standard Raman tensor $\chi_{k l}^{R}$ and the electrostrictive force $\pi_{k l}^{R}$. Equation (1) is valid only if $R_{k l}$ does not depend on frequency. In absorbing media, such as semimetals and semiconductors, the Raman tensor $R_{k l}$ depends on frequency and $F(t)$ becomes a function of the frequency. According to the theoretical work for the electrostrictive force $\pi_{k l}^{R}$ as the generation mechanism, assuming that the dielectric function $\varepsilon(\omega)$ varies slowly within the spectral width of the pump pulse, the Fourier component of the driving force $F(t)$ is expressed as follows: ${ }^{20}$

$$
F(\Omega) \propto\left[\frac{d \operatorname{Re}(\varepsilon)}{d \omega}+2 i \frac{\operatorname{Im}(\varepsilon)}{\Omega}\right] \int_{-\infty}^{+\infty} e^{i \Omega t}|E(t)|^{2} d t .
$$

Here $E(t)$ is the electric field of the pump pulse, i.e., $|E(t)|^{2}$ corresponds to the intensity of the pump light $|E(t)|^{2}=I(t)$. The real part of $\varepsilon$ leads to $F(t) \propto|E(t)|^{2}$, which is impulsive and gives $Q(t) \propto \sin \Omega_{0} t$, whereas the imaginary part of $\varepsilon$ leads to $F(t) \propto \int_{-\infty}^{t}\left|E\left(t^{\prime}\right)\right|^{2} d t^{\prime}$, which is displacive in character and gives $Q(t) \propto \cos \Omega_{0} t$. Since $|d \operatorname{Re}(\varepsilon) / d \omega| \ll \operatorname{Im}(\varepsilon) / \Omega_{0}$ with $\Omega_{0}$ being the phonon frequency, imaginary part of the dielectric function dominates the driving force. Thus we expect that the coherent optical phonon generated through resonant ISRS exhibits a displacive behavior. Solving Eq. (1) for the undamped harmonic condition $(\Gamma=0)$ yields the coherent phonon amplitude, ${ }^{20}$

$$
Q_{0} \approx \frac{\operatorname{Im}(\varepsilon) N v_{c} \Xi_{0}}{4 \pi \hbar \Omega_{0}^{2}} \int_{-\infty}^{+\infty} e^{i \Omega_{0} t}|E(t)|^{2} d t,
$$

where $\Xi_{0}$ is the deformation potential. According to Eq. (3), the amplitude of the coherent phonon depends on the imaginary part of the dielectric function as well as the pump intensity $I(t)$ if the temperature is constant, so that thermal expansion and anharmonicity of the crystal lattice, which result in modification of $v_{c}$ and $\Omega_{0}$, are negligible.

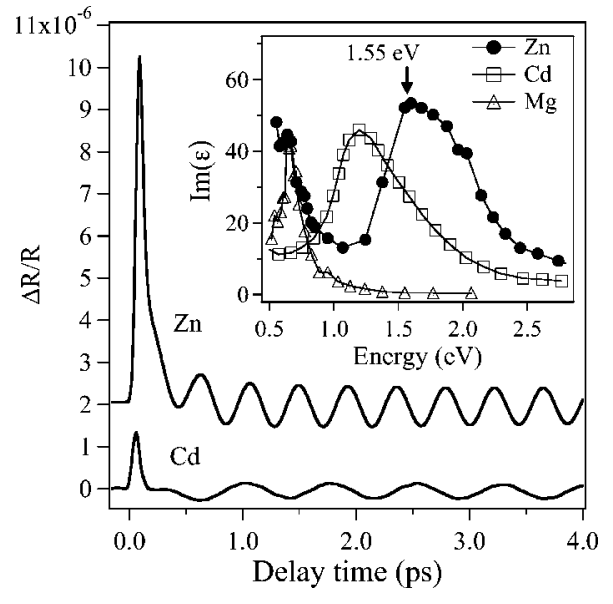

FIG. 2. Transient reflectivity change obtained for $\mathrm{Zn}$ and $\mathrm{Cd}$ at the lattice temperature $7 \mathrm{~K}$. The inset shows the imaginary part of the dielectric function $\operatorname{Im}(\varepsilon)$ for $\mathrm{Zn}$ (closed circles), Cd (open squares), and $\mathrm{Mg}$ (open triangles) (Ref. 27).

\section{EXPERIMENTAL RESULTS AND ANALYSIS}

Figure 2 shows the time-resolved TR signal $(\Delta R / R)$ obtained for $\mathrm{Zn}$ and $\mathrm{Cd}$ at $7 \mathrm{~K}$ with the pump fluence of $F_{\text {pump }}=9.2 \mu \mathrm{J} / \mathrm{cm}^{2}$. The response of the reflectivity change consists of two components. One is the initial transient nonoscillatory response because of excitation and relaxation of nonequilibrium electrons (fast electronic response), which decays in a few hundred femtoseconds. Since the interband electronic transition near the $L$ point occurs at around $800 \mathrm{~nm}(1.55 \mathrm{eV}),{ }^{27}$ this interband transition dominates generation of nonequilibrium electron distribution in $\mathrm{Zn}$.

The second component is the oscillatory signal due to generation of the coherent lattice vibration (coherent phonon response). The time period of the observed coherent oscillation is several hundred femtoseconds, corresponding to the bulk $E_{2 g}$ optical phonon mode as discussed below. It is to be noted that in addition to $\mathrm{Zn}$ and $\mathrm{Cd}$ samples, we tried to measure coherent optical phonons also in alkaline-earth metal Mg (0001), however we observed only very weak electronic response and no oscillations in the TR signal (not shown). The amplitude of the coherent optical phonon is significantly larger in $\mathrm{Zn}$ than that in $\mathrm{Cd}$. This suggests that the interband electronic transition, which contributes to the imaginary part of the dielectric function $\operatorname{Im}(\varepsilon)$ as shown in the inset of Fig. 2, governs the excitation of the coherent optical phonon, although according to Eq. (3) the phonon amplitude depends also on the deformation potential. In fact, at the laser energy of $1.55 \mathrm{eV}, \operatorname{Im}(\varepsilon)$ shows a dominant peak for $\mathrm{Zn}$, whereas it shows a weak shoulder for $\mathrm{Cd}$. For $\mathrm{Mg}$, however, $\operatorname{Im}(\varepsilon)$ is almost zero, supporting the suggestion that this mechanism is indeed the main driving force.

Hereafter we will focus on dynamics of the coherent phonon response and fast electronic response in $\mathrm{Zn}$ because the signal amplitude is much larger than that in $\mathrm{Cd}$ and therefore it is possible to make precise analysis of the time domain signal.

\section{A. Coherent phonon response}

Figure 3 shows the time-resolved TR signal $(\Delta R / R)$ obtained on $\mathrm{Zn}$ at various temperatures at the constant pump 


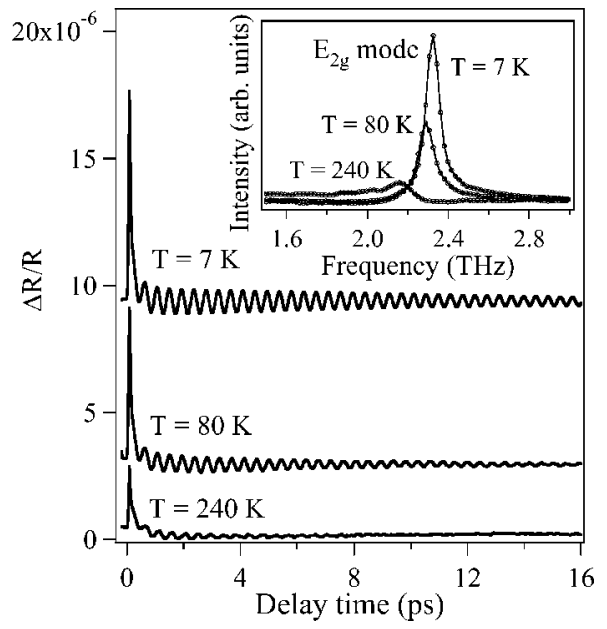

FIG. 3. Transient reflectivity change obtained in $\mathrm{Zn}$ at various lattice temperatures. The inset shows the Fourier transformed spectra of the time-domain data.

fluence of $9.2 \mu \mathrm{J} / \mathrm{cm}^{2}$. The frequency of the coherent optical phonon observed in the Fourier transformed (FT) spectra (see inset of Fig. 3) is $2.32 \mathrm{THz}$ at $7 \mathrm{~K}$, being in excellent agreement with that of the bulk $E_{2 g}$ mode observed by Raman scattering. ${ }^{21,28}$ The Fourier transformed spectra exhibit a redshift of the peak frequency and broadening of the linewidth of the $E_{2 g}$ mode as the temperature increases. The coherent phonon signal in time domain, not including ultrafast electronic response appearing at $t \leqslant 600 \mathrm{fs}$, was fitted by a damped harmonic oscillator; $A e^{-\Gamma t} \cos \left(\omega_{E_{2 g}} t+\phi_{0}\right)$, and in this way the amplitude $A$, the decay rate (damping) $\Gamma$, the frequency $\omega_{E_{2 g}}$, and the initial phase $\phi_{0}$ are extracted.

\section{Temperature dependence of damping and frequency of the $E_{2 g}$ mode}

In general, damping of the coherent phonon is governed by population decay (inelastic scattering) and/or pure dephasing (elastic scattering). In semimetal and semiconductor crystals, the decay process of the coherent phonon is dominated by the population decay due to anharmonic phonon-phonon coupling, rather than pure dephasing. ${ }^{29,30}$ The anharmonic decay rate depends strongly on the lattice temperature, while the pure dephasing, e.g., e-ph scattering, depends in metals on the hot electron density. ${ }^{16}$ In order to examine the decay process of the coherent optical phonon, temperature dependence of the decay rate at the constant pump fluence, i.e., constant hot electron density, is measured.

Figure 4 shows the decay rate of the coherent optical phonon as a function of the lattice temperature. The decay rate increases upon increasing the temperature. This behavior is well explained by the anharmonic decay model ${ }^{29}$ in which the optical phonon decays into the two acoustic phonons with half the frequency of the optical mode and with opposite wave vectors, ${ }^{29,30}$

$$
\Gamma=\Gamma_{0}\left[1+\frac{2}{\exp \left[\left(\hbar \Omega_{0} / 2\right) / k_{B} T\right]-1}\right] .
$$

Here $\Gamma_{0}$ is the effective anharmonicity as the fitting parameter and $k_{B}$ the Boltzmann constant. $\Gamma_{0}$ is determined to be

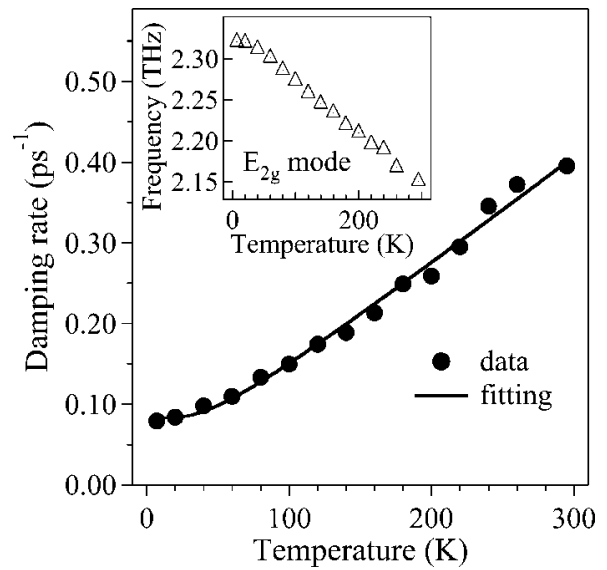

FIG. 4. Decay rate of the $E_{2 g}$ mode together with the frequency (inset) as a function of the lattice temperature. The solid line represents the fit to the data using Eq. (4).

$0.06 \mathrm{ps}^{-1}$. The good agreement of the time domain data with the anharmonic decay model indicates that the damping of the coherent $E_{2 g}$ mode in $\mathrm{Zn}$ is due to anharmonic phononphonon coupling (population decay). The frequency of the $E_{2 g}$ mode decreases as the temperature increases as shown in the inset of Fig. 4. This temperature dependence is qualitatively in good agreement with the anharmonic frequency shift observed by Raman-scattering measurements. ${ }^{28}$ Such a frequency shift due to the lattice anharmonicity was also observed in III-V semiconductors, which was reproduced by $a b$ initio calculations including various anharmonic contributions (thermal expansion, third-, and fourth-order anharmonicity). ${ }^{31}$

\section{Temperature dependence of the amplitude of the $E_{2 g}$ mode}

As shown in Fig. 5(a), the amplitude of the coherent $E_{2 g}$ phonon significantly decreases upon increasing the lattice temperature. The decrease in the amplitude of the coherent optical phonon at higher temperatures is contrary to the temperature dependence of the Raman intensity, ${ }^{21,32}$ confirming that the amplitude of the nonequilibrium coherent phonon is not determined by Bose-Einstein statistics
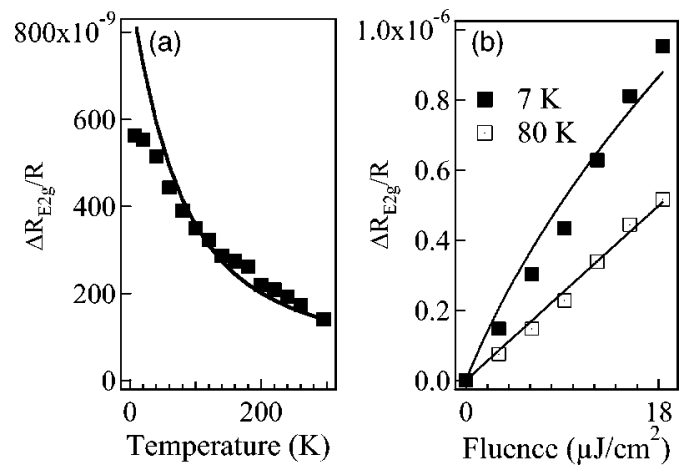

FIG. 5. (a) Amplitude of the coherent $E_{2 g}$ phonon as a function of lattice temperature. (b) Amplitude of the coherent $E_{2 g}$ phonon vs the pump fluences at the two typical temperatures. The solid curves are the fit to the data using Eq. (12). 


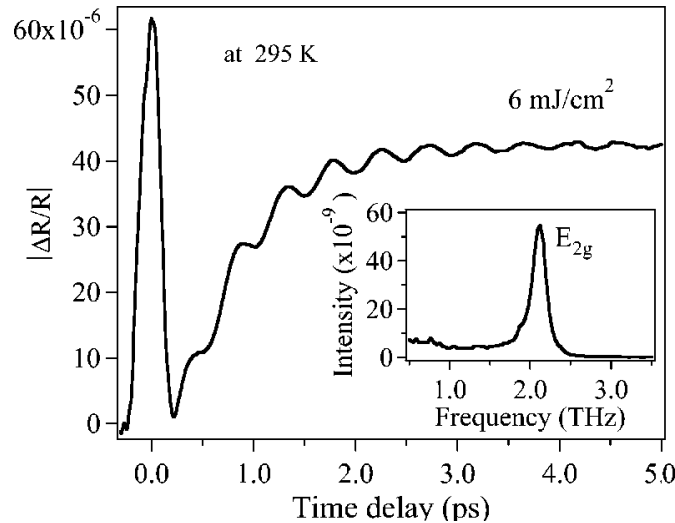

FIG. 6. Transient reflectivity change observed at $6 \mathrm{~mJ} / \mathrm{cm}^{2}$ at $295 \mathrm{~K}$. The inset shows FT spectrum of the time-domain data, showing the coherent $E_{2 g}$ mode.

$n_{k}=1 /\left[\exp \left(\hbar \Omega_{0} / k_{B} T\right)-1\right]$, which shows an increase in the phonon occupation number at higher temperatures. The very close behavior of the temperature dependence of the phonon and electronic [see below in Fig. 9(a)] responses suggests that the amplitudes of the fast electronic and the coherent phonon responses have the same origin. We will discuss the temperature dependence of the amplitude of the $E_{2 g}$ mode in Sec. V.

\section{The photoexcitation dependence}

The photoexcitation intensity dependence of the coherent $E_{2 g}$ mode was investigated in the range up to $I$ $=18.4 \mu \mathrm{J} / \mathrm{cm}^{2}$. The amplitude monotonically increases with the pump fluence both at 7 and $80 \mathrm{~K}$, as shown in Fig. 5(b). On the other hand, the frequency and the damping of the coherent $E_{2 g}$ mode were independent on the pump fluence in this fluence range. The latter result shows that the damping of coherent $E_{2 g}$ phonons in $\mathrm{Zn}$ is dominated by anharmonic phonon-phonon coupling, which is determined by the lattice temperature, rather than by pure dephasing via scattering by hot electrons. ${ }^{16}$

When increasing the pump fluence by three orders of magnitude (up to $6 \mathrm{~mJ} / \mathrm{cm}^{2}$ ) using the amplified femtosecond laser system (pulse duration of $130 \mathrm{fs}$ ) the time domain signal drastically changes. Figure 6 shows transient reflectivity change observed at $6 \mathrm{~mJ} / \mathrm{cm}^{2}$. The width of the initial electronic transient response is different from those observed in Figs. 2 and 3 because the pulse duration used in Fig. 6 is much longer than that in Figs. 2 and 3. Importantly, although the pump fluence increases by $\sim 10^{3}$, the electronic response increases only by one order of magnitude, suggesting some kind of a saturation behavior. ${ }^{33}$ The frequency of the coherent $E_{2 g}$ mode in the FT spectra (the inset of Fig. 6) is $2.10 \mathrm{THz}$, which is slightly redshifted from that obtained at $9.2 \mu \mathrm{J} / \mathrm{cm}^{2}$ at $295 \mathrm{~K}, 2.15 \mathrm{THz}$. This redshift would originate from electronic softening of the lattice ${ }^{34}$ or thermal expansion due to lattice anharmonicity ${ }^{35,36}$ under high-density photoexcitation. We note that strong background signal from lattice heating arises at $t>2 \mathrm{ps}$, and therefore the effect of thermal expansion may dominate the redshift of the coherent $E_{2 g}$ mode in $\mathrm{Zn}$.

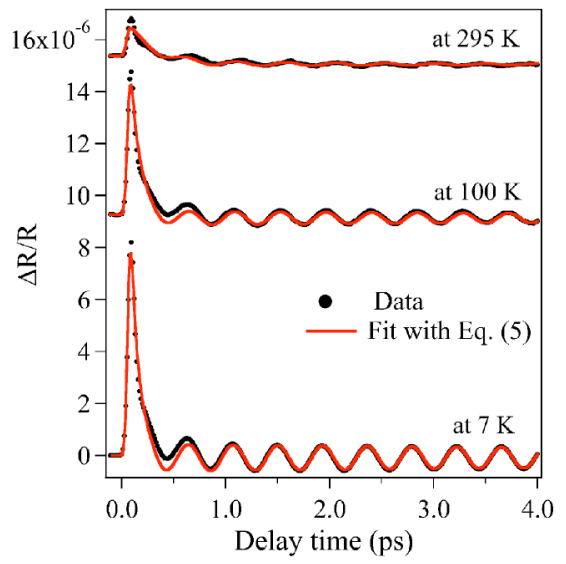

FIG. 7. (Color online) Transient reflectivity change observed for $\mathrm{Zn}$ at 7,100 , and $295 \mathrm{~K}$. The closed circles are experimental data, and the solid curve is the fitting with Eq. (5).

\section{B. Fast electronic response}

The subpicosecond electronic response measured at several temperatures is presented in Fig. 7. In order to fit the time domain data and to obtain the amplitude and relaxation time of the fast electronic transient, we utilize a linear combination of a damped harmonic oscillator and a single exponential decay function

$$
\frac{\Delta R(t)}{R}=H(t)\left[A e^{-\Gamma t} \cos \left(\omega_{E_{2 g}} t+\phi_{0}\right)+B e^{-t / \tau_{q}}+\text { const }\right] .
$$

Here $H(t)$ is the Heaviside function convoluted with Gaussian to account for the finite time resolution, while $B$ and $\tau_{q}$ are the amplitude and relaxation time of the fast electronic transient, respectively. In simple metals, such as $\mathrm{Au}, \mathrm{Ag},{ }^{9}$ ultrafast transient is usually attributed to the electron-phonon thermalization, which is treated by the so-called twotemperature model (TTM). The main idea of the TTM is that because of the fact that electron-electron thermalization is much faster than electron-phonon thermalization, electrons quickly (in tens of femtoseconds) thermalize to a temperature $T_{e}$, which can be much higher than the lattice temperature $T_{l}$, resulting in Fermi-level smearing. In the next stage, electrons thermalize with lattice in a characteristic electronphonon thermalization time, which is in metals typically in the $100 \mathrm{fs}-1 \mathrm{ps}$ range. Since after tens of femtoseconds, when electrons have already thermalized, the changes in the occupied electronic density of states are limited to energies of $k_{B} T_{e}$ near Fermi level, the photoinduced reflectivity dynamics tracks the time evolution of the electronic temperature. The TTM is given by the set of two coupled heat equations $^{37,38}$

$$
C_{e}\left(T_{e}\right) \frac{\partial T_{e}}{\partial t}=-g\left(T_{e}-T_{l}\right)+S(z, t)
$$




$$
C_{l} \frac{\partial T_{l}}{\partial t}=g\left(T_{e}-T_{l}\right)
$$

where $C_{e}\left(T_{e}\right)$ and $C_{l}$ are the respective heat capacities of electrons and lattice, $g\left[=g\left(T_{l}\right)\right]$ is the $e$-ph coupling function and $S(z, t)$ describes the absorbed energy, where $z$ is the depth coordinate. In the pump-probe experiment $S(z, t)$ has a Gaussian temporal shape. In the limit of weak perturbation (when change in the electronic temperature is small compared to the initial temperature) the relaxation of the electronic temperature is exponential with $e$-ph thermalization time $^{3}$

$$
\tau_{e-p h}=\frac{1}{g} \frac{C_{e} C_{l}}{C_{e}+C_{l}} .
$$

$g\left(T_{l}\right)$ is in the linear response limit particularly simple in the case of simple metals where the electron bandwidth is much larger than the Debye temperature $\Theta_{D}$, and the Debye model of the electron-phonon coupling can be used. In this case $g(T)=d G(T) / d T$, where 9,39

$$
G(T)=4 g_{\infty}\left(\frac{T}{\Theta_{D}}\right)^{5} \int_{0}^{\Theta_{D} / T} \frac{x^{4} d x}{e^{x}-1} .
$$

Given that, the $T$-dependence of $\tau_{e-p h}$ [Eq. (8)] is completely determined by the $e$ - $p h$ coupling constant $\left(g_{\infty}\right), \Theta_{D}, C_{e}(T)$, and $C_{l}(T)$. Since the lattice specific heat $C_{l}$ is a factor $10^{2}$ larger than the electronic specific heat $\left(C_{l} \gg C_{e}\right)$ in a wide temperature range, ${ }^{9}$ and since the electron heat capacity $C_{e}$ $=\gamma T_{e} \sim \gamma T_{l}$ (when change in the electronic temperature is small compared to the initial temperature), the $e$ - $p h$ thermalization time $\tau_{e-p h}$ is given by

$$
\tau_{e-p h} \approx \frac{C_{e}}{g\left(T_{l}\right)}=\frac{\gamma T_{l}}{g\left(T_{l}\right)},
$$

For $T_{l} \ll \Theta_{D}$ the function $g\left(T_{l}\right)$ varies $T_{l}^{4}$ and for $T_{l} \geqslant \Theta_{D}$ the function $g\left(T_{l}\right)$ becomes constant $\left(g_{\infty}\right) .{ }^{9}$ Since $\Theta_{D}=234 \mathrm{~K}$ for $\mathrm{Zn}$, TTM predicts $\tau_{e-p h} \sim T_{l}^{-3}$ at $T_{l} \leqslant \Theta_{D} / 5$ and $\tau_{e-p h} \sim T_{l}$ at $T_{l} \geqslant \Theta_{D} / 5.39$

The relaxation time of the subpicosecond transient in $\mathrm{Zn}$ obtained by fitting the data with Eq. (5) is shown in Fig. 8 as the function of lattice temperature. The relaxation time monotonically increases from $75 \pm 30$ fs to $260 \pm 30 \mathrm{fs}$ as the temperature increases, similar to the behavior obtained for noble metals. ${ }^{9}$ The behavior follows the prediction of the TTM at temperatures above $50 \mathrm{~K}$, whereas no upturn in relaxation time is observed at low temperatures. The absence of upturn in relaxation time at low temperatures is common in metals, ${ }^{9}$ and is attributed to the fact that the basic assumption of the TTM that $e$ - $e$ thermalization time is fast compared to the $e$-ph thermalization fails at low temperatures. Therefore a nonthermal electron model (NEM) needs to be introduced that accounts for the low-temperature saturation of relaxation time ${ }^{9,39}$ — the analysis using a NEM is beyond the scope of this paper. By fitting the temperature dependence of the relaxation time with Eq. (10) we obtain the value of the $e$-ph coupling constant $g_{\infty}=6.4 \times 10^{6} \mathrm{Wm}^{-3} \mathrm{~K}^{-1}$, which is the same order of magnitude as in other simple metals. ${ }^{6}$

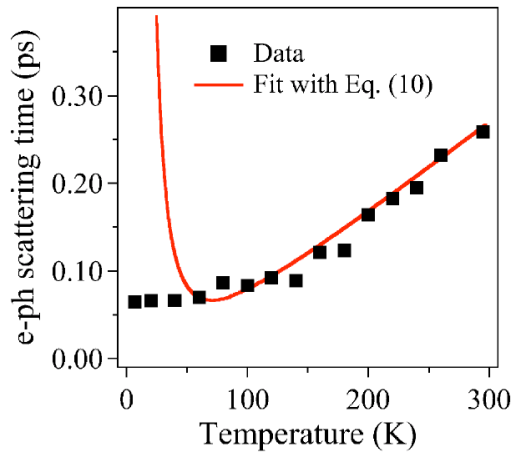

FIG. 8. (Color online) Temperature dependence of the electronphonon thermalization time $\left(\tau_{q}\right)$. The closed squares are experimental data, whereas the solid curve is a fit to the data with the TTM model, described by Eq. (10).

\section{Temperature dependence of electronic transient}

As shown above, the temperature dependence of the relaxation time follows the prediction of the thermomodulation scenario, where the data above $50 \mathrm{~K}$ can be well fitted using the standard TTM. On the other hand, the amplitude of the fast electronic response, $B$ in Eq. (5), also shows pronounced temperature dependence as shown in Fig. 9(a). Moreover the same temperature dependence is obtained also for the amplitude of the oscillatory transient due to the photoexcited coherent $E_{2 g}$ phonon, suggesting that the amplitudes of the fast electronic and the coherent phonon responses have the same origin.

It should be noted that the temperature dependence of the amplitude of the photoinduced reflectivity transient has thus far not been studied for the case of simple metals. Below we derive the temperature dependence of the transient amplitude $B$ as would be expected in the case of the thermomodulation mechanism and show extremely good agreement with the data.

To account for the temperature dependence of $B$ in Eq. (5), we assume that the relaxation processes after excitation
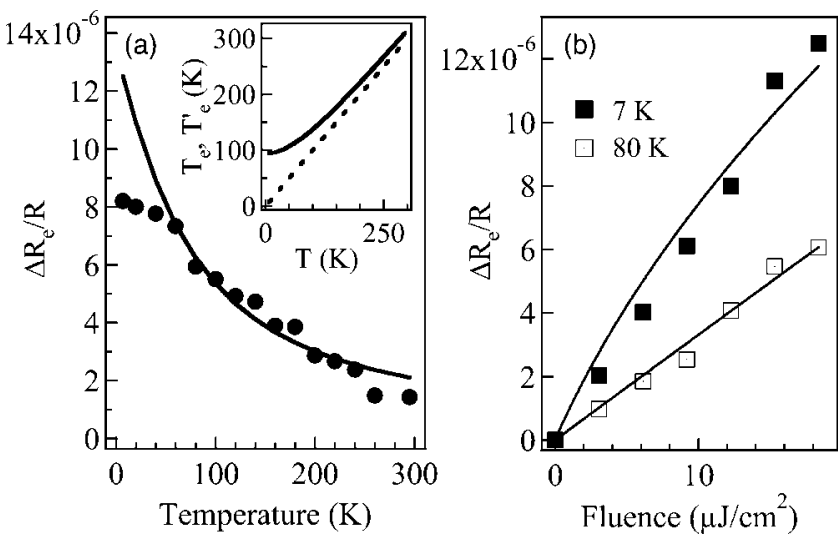

FIG. 9. (a) Amplitude of the fast electronic transient as a function of temperature. Inset shows the corresponding initial ( $T_{e}$-dashed line $)$ and final $\left(T_{e}^{\prime}\right.$-solid line $)$ electronic temperatures as a function of temperature. (b) Amplitude of the fast electronic transient vs the pump fluences at the two typical temperatures. The solid curves are the fit to the data using Eq. (12). 
with short laser pulse follow the TTM, i.e., electron-electron thermalization is much faster than $e$ - $p h$ thermalization. Furthermore, we assume that the changes in the reflectivity at optical frequency $(1.55 \mathrm{eV})$ are due to photoinduced absorption, with photoinduced quasiparticles (in the energy range of $k_{B} T_{e}$ around the Fermi energy) as initial states and with final unoccupied states well above the Fermi energy. ${ }^{40,41} \mathrm{We}$ should note that similar arguments have been successfully applied for the case of optical pump-probe spectroscopy in superconductors ${ }^{42}$ and charge density waves. ${ }^{43}$ Therefore, using the Fermi golden rule, the amplitude of the transient is proportional to the change in the occupied electronic density of states near $E_{F}$, i.e., the amplitude of the photoinduced transient should be proportional to the photoinduced quasiparticle density $n_{p}$. In the limit when temperature is much lower than the Fermi energy, which is the case here, the number density of the electron-hole pairs $n$ is in the Landau Fermi liquid exactly proportional to temperature, $n \propto T$. We assume that after photoexcitation all the absorbed energy initially goes in the electronic subsystem, and the electrons are described by the increased electronic temperature $T_{e}^{\prime}$. Following this one obtains

$$
B \propto n_{p}=n_{T_{e}^{\prime}}-n_{T_{e}} \propto T_{e}^{\prime}-T_{e},
$$

where $n_{T_{e}}$ is the quasiparticle density at the initial temperature $T_{e}$, while $n_{T_{e}^{\prime}}$ is the quasiparticle density after electrons have thermalized to final temperature $T_{e}^{\prime}$. Taking into account that the electronic specific heat $C_{e}=\gamma T_{e}$, where $\gamma$ is the Sommerfeld constant and using the energy conservation law it follows:

$$
B \propto T_{e}^{\prime}-T_{e}=\sqrt{T_{e}^{2}+2 U_{l} / \gamma}-T_{e},
$$

where $U_{l}$ is the absorbed energy intensity.

There are several important implications of Eq. (12). First, the amplitude of the transient is maximum at low temperatures and decreasing as the sample temperature is increasing. Second, at low temperatures the model predicts a sublinear excitation intensity dependence, while at high temperatures the dependence becomes more and more linear.

In Fig. 9 we show that the model, indeed, well reproduces the observed temperature dependence. Here $U_{l} / \gamma$ was taken as a fitting parameter since the estimated absolute value of $U_{l}$ is subject to some uncertainty (possible errors in the spot size, reflectance, and the optical penetration depth may give rise to substantial error). Importantly, the final temperature $T_{e}^{\prime}$ obtained from the same fit [shown in the inset to Figure 9(a)] matches well the estimate given in Ref. 23.

Although very good agreement with the temperature dependence of $B$ is obtained at temperatures higher than $\sim 50 \mathrm{~K}, B$ shows saturation below $50 \mathrm{~K}$, while the model predicts further increase in the amplitude. We ascribe this discrepancy to the fact that at low temperatures the TTM fails, since $e-e$ thermalization time becomes comparable or even longer than the $e$-ph thermalization time. ${ }^{39}$

Figure 9(b) presents the excitation intensity dependence of amplitude measured at 7 and $80 \mathrm{~K}$, together with the fit using Eq. (12) using the same value of $U_{l} / \gamma$ as extracted from the fit to the temperature-dependence data. As seen, the model predicts the sublinear dependence at $7 \mathrm{~K}$, which is not observed in this intensity range, while good agreement with $80 \mathrm{~K}$ data is obtained. We believe that for the same reasons that the temperature dependences of relaxation time and amplitude at low temperatures do not follow the TTM prediction; the fact that electrons are non thermal on this time scale can give rise to the absence of the sublinear intensity dependence at low temperatures.

\section{DISCUSSION}

According to the model by Stevens et al. ${ }^{20}$ under the condition that the lattice temperature is constant, the amplitude of the coherent phonon depends on the imaginary part of the dielectric function $\operatorname{Im}(\varepsilon)$ as well as the pump intensity $I(t)$ [see Eq. (3)]. Here we review the temperature dependence of the coherent phonon amplitude in $\mathrm{Zn}$ in terms of Eq. (3). When the lattice temperature increases, thermal expansion occurs and the volume $v_{c}$ increases. This effect will increase the phonon amplitude according to Eq. (3). The phonon frequency decreases for $\approx 8 \%$ with increasing the temperature from 7 to $295 \mathrm{~K}$, suggesting an amplitude increase [Eq. (3)] by $\approx 16 \%$ contrary to our experimental results, which shows a decrease in the coherent phonon amplitude as the temperature increases. The deformation potential $\Xi_{0}$ can be taken as nearly constant through the entire temperature range in the present study. ${ }^{44}$ Thus, any parameters other than $\operatorname{Im}(\varepsilon)$ in Eq. (3) cannot account for the temperature dependence of the coherent phonon amplitude.

The initial phase of the coherent optical phonon is estimated to be $5^{\circ} \pm 5^{\circ}$ at all temperatures from the fitting the oscillatory part with the single damped harmonic oscillator, indicating that the coherent phonon oscillation follows cosine behavior. The cosine phase is consistent with the prediction from Stevens' model [see Eq. (2)], where the driving force of resonant ISRS has displacive character. Further argument for the displacive nature of the observed transient is the strong correlation between the photoinduced quasiparticle density and the amplitude of the coherent optical phonon observed in both temperature and fluence dependences. For example, in Fig. 5 we use the same fitting function as used for describing the temperature dependence of the electric transient. Fitting of the coherent phonon amplitude versus both the temperature and the fluence using Eq. (12) is surprisingly good. As discussed in Sec. III, the driving force $F(t) \propto \int_{-\infty}^{t}\left|E\left(t^{\prime}\right)\right|^{2} d t^{\prime}$ is the Heaviside function convoluted with Gaussian and causes displacive behavior for the excitation of Raman active mode through resonant ISRS process. Because $\operatorname{Im}(\varepsilon)$ is directly related to photo excitation of nonequilibrium electrons, quasiparticle density should follow $\operatorname{Im}(\varepsilon): n_{p} \propto \operatorname{Im}(\varepsilon)$. Thus under the resonant condition, $Q_{0}$ $\propto \operatorname{Im}(\varepsilon)$ and $n_{p} \propto \operatorname{Im}(\varepsilon)$.

\section{CONCLUSIONS}

We have investigated ultrafast dynamics of coherent optical phonons and nonequilibrium electrons in transition metal $\mathrm{Zn}$ and $\mathrm{Cd}$ using a femtosecond pump-probe technique. The optical response in time domain is well fitted by linear com- 
bination of a damped harmonic oscillation due to excitation of coherent $E_{2 g}$ phonon and a subpicosecond electronic transient. Dynamics of both the nonequilibrium electron distribution and the photoexcited coherent $E_{2 g}$ phonon were observed in a wide temperature range from 7 to $295 \mathrm{~K}$. The amplitudes of both the coherent optical phonon and electronic transient show pronounced temperature dependencies. Based on the fact that the relaxation time of the fast electronic transient closely follows the prediction of the thermomodulation scenario we analyze the relaxation dynamics in terms of the two-temperature model. Good agreement with the model is obtained between $50 \mathrm{~K}$ and room temperature, while below $50 \mathrm{~K}$ the model is found to fail similar to studies on simple metals, such as $\mathrm{Au}$ or Ag. ${ }^{9}$ Below $50 \mathrm{~K}$ a nonthermal electron model ${ }^{9}$ needs to be applied.

In order to account for the temperature dependence of the amplitude we developed a model assuming the photoinduced absorption mechanism, where the signal amplitude is proportional to the photoinduced change in the quasiparticle density. The model was found to account for the observed temperature dependence over wide temperature range. Importantly, the model predicts a sublinear photoexcitation dependence at low temperatures, which was not observed in the excitation range studied. This discrepancy may be due to the failure of the two-temperature model at low temperatures. The fact that the amplitude of the coherent $E_{2 g}$ phonon follows the temperature dependence of the amplitude of the fast electronic transient suggests that under the resonant condition both electronic and phononic responses are proportional to the change in the dielectric function.
The damping and the frequency of the coherent $E_{2 g}$ phonon were also found to depend on the lattice temperature, which was explained by anharmonic phonon-phonon coupling, rather than pure dephasing, and by anharmonic frequency shift, respectively. There was no dependence of the frequency and the damping of the $E_{2 g}$ phonon on the pump fluence, suggesting that pure dephasing due to scattering with hot electrons is negligibly small. When increasing the pump fluence up to the millijoule per centimeter-squared $\left(\mathrm{mJ} / \mathrm{cm}^{2}\right)$ range, the redshift of the coherent $E_{2 g}$ mode frequency was observed, which was ascribed to thermal expansion. Since the $E_{2 g}$ mode in $\mathrm{Zn}$ may be sensitive to highly anisotropic compressional behavior of $c / a$ axial ratio in $\mathrm{Zn}$ crystal (hcp structure), as observed by Raman scattering under pressure, controlling the amplitude and the frequency of the coherent $E_{2 g}$ phonon will be useful for the study of the gradual anisotropic $\rightarrow$ isotropic transition occuring in $\mathrm{Zn} .{ }^{45}$

\section{ACKNOWLEDGMENTS}

The authors acknowledge Hrvoje Petek and Viktor Kabanov for helpful comments. This work was supported by a Grant-in-Aid for Scientific Research from the Ministry of Education, Culture, Sports, Science, and Technology of Japan under Grant No. KAKENHI-15740188 and No. KAKENHI-15035219. The authors acknowledge partial financial support from NIMS Research Funds and the Crossover Research Fund of MEXT, Japan.
*Electronic address: hase.muneaki@nims.go.jp

${ }^{1}$ D. Pines, in Elementary Excitations in Solids (Benjamin, New York, 1963), Chap. 5.

${ }^{2}$ J.-C. Diels and W. Rudolph, in Ultrashort Laser Pulse Phenomena: Fundamentals, Techniques, and Applications on a Femtosecond Time Scale, Chap. 8 (Academic, New York, 1996).

${ }^{3}$ C. K. Sun, F. Vallée, L. Acioli, E. P. Ippen, and J. G. Fujimoto, Phys. Rev. B 48, 12365 (1993).

${ }^{4}$ W. S. Fann, R. Storz, H. W. K. Tom, and J. Bokor, Phys. Rev. B 46, 13592 (1992).

${ }^{5}$ N. Del Fatti, R. Bouffanais, F. Vallée, and C. Flytzanis, Phys. Rev. Lett. 81, 922 (1998).

${ }^{6}$ J. Hohlfeld, S.-S. Wellershoff, J. Güdde, U. Conrad, V. Jäanke, and E. Mattias, Chem. Phys. 251, 237 (2000).

${ }^{7}$ S. I. Anisimov, B. L. Kapeliovich, and T. L. Perel'man, Sov. Phys. JETP 39, 375 (1974).

${ }^{8}$ H. Hirori, T. Tachizaki, O. Matsuda, and O. B. Wright, Phys. Rev. B 68, 113102 (2003).

${ }^{9}$ R. H. M. Groeneveld, R. Sprik, and A. Lagendijk, Phys. Rev. B 51, 11433 (1995).

${ }^{10}$ O. B. Wright and K. Kawashima, Phys. Rev. Lett. 69, 1668 (1992).

${ }^{11}$ O. B. Wright, Phys. Rev. B 49, 9985 (1994).

${ }^{12}$ I. Bozovic, M. Schneider, Y. Xu, R. Sobolewski, Y. H. Ren, G. Lüpke, J. Demsar, A. J. Taylor, and M. Onellion, Phys. Rev. B 69, 132503 (2004).
${ }^{13}$ A. Melnikov, I. Radu, U. Bovensiepen, O. Krupin, K. Starke, E. Matthias, and M. Wolf, Phys. Rev. Lett. 91, 227403 (2003).

${ }^{14}$ H. J. Zeiger, J. Vidal, T. K. Cheng, E. P. Ippen, G. Dresselhaus, and M. S. Dresselhaus, Phys. Rev. B 45, 768 (1992).

${ }^{15}$ U. Bovensiepen, A. Melnikov, I. Radu, O. Krupin, K. Starke, M. Wolf, and E. Matthias, Phys. Rev. B 69, 235417 (2004).

${ }^{16}$ K. Watanabe, N. Takagi, and Y. Matsumoto, Phys. Rev. Lett. 92, 057401 (2004)

${ }^{17}$ G. A. Garrett, T. F. Albrecht, J. F. Whitaker, and R. Merlin, Phys. Rev. Lett. 77, 3661 (1996).

${ }^{18}$ C. Guo, G. Rodriguez, A. Lobad, and A. J. Taylor, Phys. Rev. Lett. 84, 4493 (2000).

${ }^{19}$ M. U. Wehner, M. H. Ulm, D. S. Chemla, and M. Wegener, Phys. Rev. Lett. 80, 1992 (1998).

${ }^{20}$ T. E. Stevens, J. Kuhl, and R. Merlin, Phys. Rev. B 65, 144304 (2002).

${ }^{21}$ W. B. Grant, H. Schulz, S. Hüfner, and J. Pelzl, Phys. Status Solidi B 60, 331 (1973).

${ }^{22}$ Assuming hyperbolic secant function for the laser pulse, we obtain net pulse duration $\Delta \tau$ from the pulse duration of AC $\Delta \tau_{A C}$, where $\Delta \tau=\Delta \tau_{A C} \times 0.648$ (See Ref. 2).

${ }^{23}$ The maximum electron temperature rise is estimated using a function of $\Delta T_{e}^{m}=\left(T_{l}^{2}+2 U_{l} / \gamma\right)^{1 / 2}-T_{l}$ (see Ref. 9), where $U_{l}$ is the pump energy density, the lattice temperature $T_{l}$ is set to be $300 \mathrm{~K}$, and the Sommerfeld constant $\gamma=64 \mathrm{~J} \mathrm{~m}^{-3} \mathrm{~K}^{-2}$ for $\mathrm{Zn}$.

${ }^{24}$ A. Arbouet, C. Voisin, D. Christofilos, P. Langot, N. Del Fatti, F. 
Vallée, J. Lermé, G. Celep, E. Cottancin, M. Gaudry, M. Pellarin, M. Broyer, M. Maillard, M. P. Pileni, and M. Treguer, Phys. Rev. Lett. 90, 177401 (2003).

${ }^{25}$ T. Dekorsy, G. C. Cho, and H. Kurz, in Light Scattering in Solids VIII, edited by M. Cardona and G. Güntherodt (Springer-Verlag, Berlin, 2000), Chap. 4.

${ }^{26}$ Yong-Xin Yan, E. B. Gamble, Jr., and K. A. Nelson, J. Chem. Phys. 83, 5391 (1985).

${ }^{27}$ P. Grosse and W. Richter, in Landolt-Börnstein, edited by O. Madelung, New Series, Vol. 17 (Springer, Berlin, 1983).

${ }^{28}$ H. Schultz and S. Hüfner, Solid State Commun. 20, 827 (1976).

${ }^{29}$ F. Vallée, Phys. Rev. B 49, 2460 (1994).

${ }^{30}$ M. Hase, K. Mizoguchi, H. Harima, S. I. Nakashima, and K. Sakai, Phys. Rev. B 58, 5448 (1998).

${ }^{31}$ A. Debernardi, Solid State Commun. 113, 1 (2000).

${ }^{32}$ M. Cardona, Chap. 5, in Raman Scattering in Materials Science, edited by W. H. Weber and R. Merlin, Springer Series in Materials Science (Springer, New York, 2000).

${ }^{33}$ M. F. DeCamp, D. A. Reis, P. H. Bucksbaum, and R. Merlin, Phys. Rev. B 64, 092301 (2001).

${ }^{34}$ S. Hunsche, K. Wienecke, T. Dekorsy, and H. Kurz, Phys. Rev. Lett. 75, 1815 (1995).

${ }^{35}$ M. Perner, S. Gresillon, J. März, G. von Plessen, J. Feldmann, J. Porstendorfer, K.-J. Berg, and G. Berg, Phys. Rev. Lett. 85, 792
(2000).

${ }^{36}$ M. Hase, M. Kitajima, S. I. Nakashima, and K. Mizoguchi, Phys. Rev. Lett. 88, 067401 (2002).

${ }^{37}$ M. I. Kaganov, I. M. Lifshitz, L. V. Tanatarov, Sov. Phys. JETP 4, 173 (1957).

${ }^{38}$ P. B. Allen, Phys. Rev. Lett. 59, 1460 (1987).

${ }^{39}$ J. Demsar, R. D. Averitt, K. H. Ahn, M. J. Graf, S. A. Trugman, V. V. Kabanov, J. L. Sarrao, and A. J. Taylor, Phys. Rev. Lett. 91, 027401 (2003); K. H. Ahn, M. J. Graf, S. A. Trugman, J. Demsar, R. D. Averitt, J. L. Sarrao, and A. J. Taylor, Phys. Rev. B 69, 045114 (2004).

${ }^{40}$ R. W. Schoenlein, W. Z. Lin, J. G. Fujimoto, and G. L. Eesley, Phys. Rev. Lett. 58, 1680 (1987).

${ }^{41}$ G. L. Eesley, Phys. Rev. Lett. 51, 2140 (1983).

${ }^{42}$ V. V. Kabanov, J. Demsar, B. Podobnik, and D. Mihailovic, Phys. Rev. B 59, 1497 (1999).

${ }^{43}$ J. Demsar, K. Biljakovic, and D. Mihailovic, Phys. Rev. Lett. 83, 800 (1999); J. Demsar, L. Forró, H. Berger, and D. Mihailovic, Phys. Rev. B 66, 041101(R) (2002).

${ }^{44}$ H. J. Zeiger, T. K. Cheng, E. P. Ippen, J. Vidal, G. Dresselhaus, and M. S. Dresselhaus, Phys. Rev. B 54, 105 (1996).

${ }^{45}$ H. Olijnyk, A. P. Jephcoat, D. L. Novikov, and N. E. Christensen, Phys. Rev. B 62, 5508 (2000). 